\title{
Higher In vitro Proliferation Rate of Rhizopus oryzae in Blood of Diabetic Individuals in Chronic Glycaemic Control Compared with Non-diabetic Individuals
}

\author{
Grace Salazar-Tamayo • Luis E. López-Jácome • Jesús Resendiz-Sanchez • \\ Rafael Franco-Cendejas · Patricia Rodriguez-Zulueta · Dora E. Corzo-León
}

Received: 13 January 2017 / Accepted: 29 June 2017/Published online: 6 July 2017

(C) The Author(s) 2017. This article is an open access publication

\begin{abstract}
Metabolic control improves outcomes associated with mucormycosis. The aim of this study was to compare the in vitro proliferation of Rhizopus oryzae in blood of individuals with and without diabetes at different glycaemic levels. Ninety-five individuals were included. Blood samples from each participant were incubated with sporangiospores of $R$. oryzae. The germination, filamentation and growth of $R$. oryzae were compared at different time points. Four groups were defined, one without (group A, $n=30$ ) and three with diabetes: group B (HbA1c $\leq 7 \%$, $N=24)$, group C (HbA1c 7.1-9\%, $N=20$ ) and group D (HbA1c $>9 \%, N=21)$. The percentage of germinated sporangiospores was higher in the group A after $6 \mathrm{~h}$ (group A 56\% \pm 3 , group B 35\% \pm 4 , group C $48 \% \pm 4$, group D $46 \% \pm 1.4, p=0.01), 12 \mathrm{~h}$ (group A $54 \% \pm 1.4$, group B $19 \% \pm 4$, group C $16 \% \pm 1$, group D $9.5 \% \pm 5, p<0.001)$ and $24 \mathrm{~h}$
\end{abstract}

Electronic supplementary material The online version of this article (doi:10.1007/s11046-017-0174-0) contains supplementary material, which is available to authorized users.

G. Salazar-Tamayo · P. Rodriguez-Zulueta

D. E. Corzo-León

Department of Infectious Diseases and Hospital

Epidemiology, Hospital General Dr Manuel Gea

González, Mexico City, Mexico

L. E. López-Jácome · R. Franco-Cendejas

Laboratory of Microbiology and Department of Infectious

Diseases, Instituto Nacional de Rehabilitación,

Mexico City, Mexico (group A $29 \% \pm 1$, group B $12 \% \pm 4$, group C $13.5 \% \pm 3.5$, group D $12 \% \pm 1, p<0.01)$. The filamentation was higher in groups with diabetes. Group B showed higher filamentation grade than group $\mathrm{A}$ at $6 \mathrm{~h}(0.4 \pm 0.04$ vs $1 \pm 0.09, p<0.001)$ and $24 \mathrm{~h}(1.6 \pm 0.05$ vs $2.1 \pm 0.1, p=0.05)$. In conclusion, $R$. oryzae proliferation was higher among diabetic individuals, including good glycaemic control, than among non-diabetic individuals.

Keywords Mucormycosis - Diabetes · Proliferation · Germination - Rhizopus oryzae

\section{Introduction}

The principal agent causing mucormycosis is Rhizopus oryzae. Mucormycosis is a lethal fungal infection affecting mainly individuals with diabetes, individuals with haematological malignancies [1-4] and individuals

\footnotetext{
J. Resendiz-Sanchez

Laboratory of Mycology, Hospital Infantil de México, Mexico City, Mexico

D. E. Corzo-León $(\bowtie)$

Aberdeen Fungal Group. MRC Centre of Medical Mycology, Wellcome Trust Strategy Award. Institute of Medical Sciences, University of Aberdeen,

Aberdeen City, Scotland, UK

e-mail: cold200781@yahoo.com
} 
with trauma [5, 6]. Chronic and acute forms of glycaemic uncontrolled diabetes have been described as main risk factors in the development of mucormycosis [7-10], and this is due to the role of glucose in modulating angioinvasion [11]. Metabolic control has shown to decrease the susceptibility to mucormycosis and to improve the survival rate associated with this infection in animal models [12, 13]. Also, it has been highlighted, by clinical guidelines and experts in the field, that metabolic control is an essential component in the management of mucormycosis when it affects diabetic populations $[14,15]$. In general, one of the recommendations in metabolic control of diabetes is to get a target of glycosylated haemoglobin ( $\mathrm{HbA1c})<7 \%$ [16]. Hence, the aim of this study was to compare the in vitro proliferation of $R$. oryzae in blood of individuals with and without diabetes. Our results showed lesser and slower in vitro proliferation of $R$. oryzae in samples of diabetic individuals and good glycaemic control in comparison with uncontrolled counterparts; however, it was still higher than the individuals without diabetes.

\section{Materials and Methods}

\section{Included Population and Blood Samples}

This project was approved by the local IRB at Hospital General Dr Manuel Gea González with the number 36-55-2015. The selection of participants was based on their previously programmed hospital check-up appointments and the period of time when this study was held. During this period, 1505 individuals were identified as potential participants but only 141 had programed an appointment for HbA1c test. Finally, 95 individuals were included, and blood samples and clinical data were obtained. After a revision of medical records to confirm the diabetes status of the selected individuals (the diagnosis of diabetes was done independently by the healthcare provider/physician as part of a regular standard of care), they were categorised into groups with and without diabetes and by HbA1c level, as shown in Fig. 1.

One blood sample was drawn from each participant in tube without anticoagulant (SST BD Vacutainer ${ }^{\circledR}$ ). All samples were subject to mechanical and freezing cellular lysis. Mechanical cellular lysis consisted of vortexing the sample for $5 \mathrm{~min}$; meanwhile, freezing cellular lysis consisted of storing the samples at $-70{ }^{\circ} \mathrm{C}$ until their use for further in vitro assays.

\section{Strains and Culture}

During the in vitro assays, a respiratory clinical isolate of $R$. oryzae was used. This isolate was identified by conventional methods and sequencing. Conventional methods consisted of morphology identification of colony and microscopy using lactophenol cotton blue stain. ITS1-2 regions were amplified and sequenced using the universal primers ITS1/ITS4 previously published elsewhere [17], nucleotide sequence accession number MF379466.

The $R$. oryzae strain was cultured and maintained in Sabouraud dextrose agar (SDA) for five days at $30{ }^{\circ} \mathrm{C}$. Following the CLSI M38A guidelines, sporangiospores suspension was collected to obtain a solution at 0.5 McFarland corresponding to $10^{5}$ sporangiospores $/ \mathrm{mL}$. Ninety-five sporangiospores suspensions from the same strain, one for each participant, were collected. Sporangiospores suspensions were kept frozen at $-70{ }^{\circ} \mathrm{C}$ until their further use.

\section{Design of the Assay}

For in vitro interaction assays, after thawing each blood sample and sporangiospores, $500 \mu \mathrm{l}$ of blood of each participant and $500 \mu \mathrm{l}$ of sporangiospores suspension (a dilution 1:2) were mixed and incubated at $30{ }^{\circ} \mathrm{C}$. Each assay was performed in duplicate.

\section{Definitions}

The growth of $R$. oryzae was evaluated at the time of the inoculation (time 0), and at 3, 6, 12 and $24 \mathrm{~h}$ of incubation. Observations were made in the same way for each one of the 95 samples. These observations were as follows: 1) number of sporangiospores and germination rate per $\mathrm{mL}, 2$ ) filamentation/hyphae formation and 3) growth in Sabouraud dextrose agar (SDA). The number of sporangiospores was estimated per $\mathrm{mL}$ using a haemocytometer, and germination rate was estimated dividing the elongated sporangiospores by the total number of sporangiospores. To evaluate the filamentation, $10 \mu \mathrm{l}$ of the sample on slide with $40 \% \mathrm{KOH}$ solution was observed using light microscopy at $40 \times$ objective. The grade of filamentation 


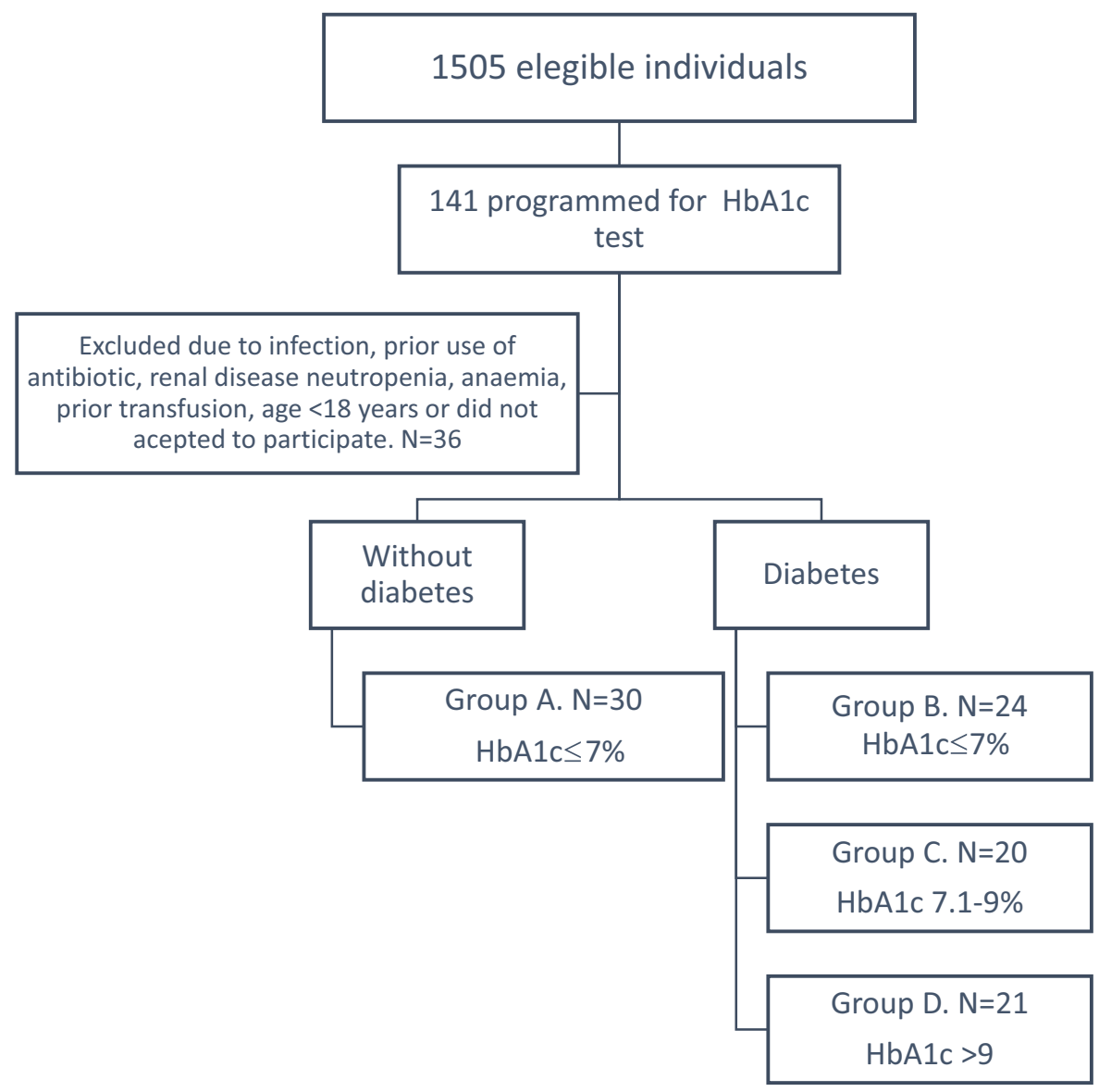

Fig. 1 Study design and categories of the study groups. The selection of the participants was done based on previously check-up appointments, and they had to have a HbA1c test programmed. The study groups were categorised depending on

was evaluated as follows: 1) Grade 1: 1-100 hyphae observed per 100 fields, 2) Grade 2: 101-200 hyphae per 100 observed fields and 3) Grade 3: $>200$ hyphae per 100 observed fields. SDA growth was measured in millimetres after culturing $10 \mu \mathrm{l}$ of each sample in a SDA after $12 \mathrm{~h}$ of incubation, as shown in Fig. 2.

\section{Statistical Analysis}

Results are presented as proportions and/or median or mean values (mean corresponding to the duplicated assays) as required. Comparisons were made with Kruskal-Wallis, one-way ANOVA, Fisher's LSD and Dunnett's tests as appropriate. $P$ value $\leq 0.05$ was considered statistically significant when two groups were compared and $p$ value $\leq 0.01$ when four groups were compared between them. Statistical tests were the HbA1c levels, group $A$ : individuals without diabetes, individuals with diabetes were group $B(\mathrm{HbAlc} \leq 7 \%)$, group $C$ (HbA1c 7.1-9\%), group D (HbA1c > 9\%)

performed using SPSS Inc 24 software (IBM Corporation, New York, USA).

\section{Results}

The 95 individuals included in this study were categorised depending on the HbA1c level. The categorisation was made after all in vitro assays were performed to be blind during the assay.

Clinical Characteristics Between Groups were not Statistically Different Except for Glycaemic Control

Age, statin use, biochemical parameters (such as creatinine levels and haematic cytometry), except 

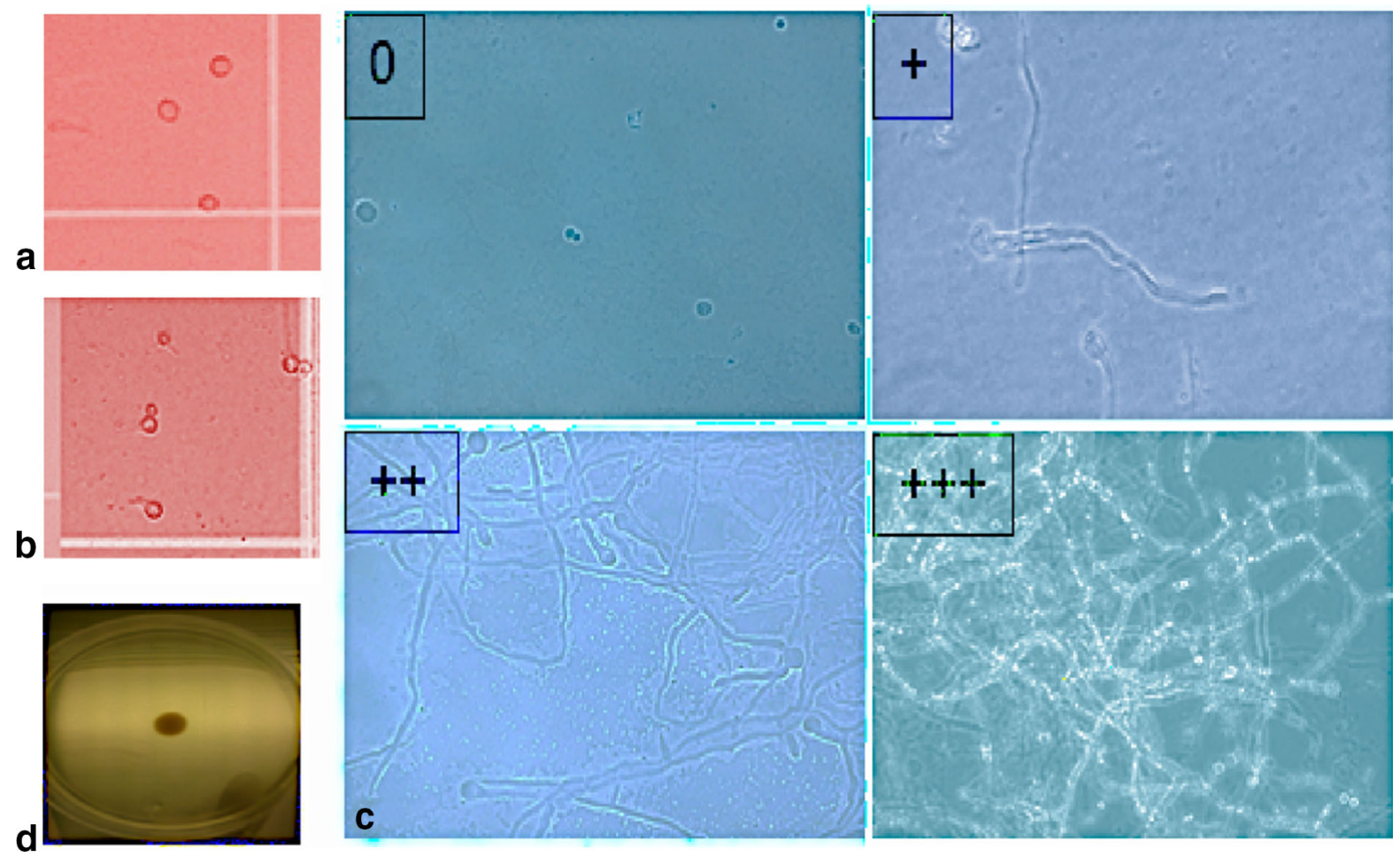

Fig. 2 Definitions of the variables used to evaluate in vitro growth of $R$. oryzae in this study. a Number of sporangiospores per $\mathrm{mL}$ (Magnification $\times 40$ ), b germination rate (elongated bodies per total of sporangiospores seen per $\mathrm{mL}$, magnification $\times 40$ ), $\mathbf{c}$ grade of filamentation/hypha formation per 100 fields

fasting glucose and $\mathrm{HbA1c}$, were not significantly different between groups as given in Table 1. Only fasting glucose and HbAlc were different between groups $(p<0.05)$. The highest fasting glucose value was $456 \mathrm{~g} / \mathrm{dL}$ and was found in one subject in group D. Acute hyperosmolar state was ruled out in this subject. Levels of $\mathrm{HbA} 1 \mathrm{c}$ were $\leq 7 \%$ for groups $\mathrm{A}$ and $\mathrm{B}$ (median, minimum-maximum values 5.7, 4-6.4\% vs $6.45,5.3-7 \%)$ and for group $\mathrm{C}$ and group $\mathrm{D}>7.1$ (median, minimum-maximum values $8,7.1-8.9 \%$ vs $10.8,9.3-15.3 \%)$.

In vitro Germination Process Started Earlier in all Groups with Diabetes but Lasts Longer in the Group Without Diabetes

The number of sporangiospores at the beginning of the assay between groups (254 vs 306 vs 334 vs 341 , $p=0.21$ ) decreased after $3 \mathrm{~h}$ of incubation (Fig. 3). Differences in the decreasing number of sporangiospores were evident between the group without
$(0=$ no hyphae in 100 fields, $+=1-100$ hyphae in 100 fields, $++=101-200$ hyphae in 100 fields, $+++=$ more than 201 hyphae in 100 fields); magnification $\times 240$, $\mathbf{d}$ growth in SDA measured in $\mathrm{Mm}$. Sporangiospores and germinated bodies were counted using an haemocytometer

diabetes compared with the groups with diabetes after $6 \mathrm{~h}$ of incubation (group A $111 \pm 2.1$ vs group B $62 \pm 5.6$, group C $36 \pm 9.8$, group D $41 \pm 5$, $p<0.001)$. In groups $\mathrm{C}$ and $\mathrm{D}$ with diabetes, levels of $\mathrm{HbA} 1 \mathrm{c}$ between 7.1-9\% and $>9 \%$, respectively, the number of sporangiospores did not differ between them (group C $36 \pm 9.8$ vs group D $41 \pm 5, p=0.8$ ); however, the number of sporangiospores in these groups decreased higher and faster than in the group B with HbA1c level in glycaemic control $(\mathrm{HbA} 1 \mathrm{c}<7 \%)$. After $6 \mathrm{~h}$ of incubation, between groups $\mathrm{A}$ (without diabetes) and $\mathrm{B}$, the decrease in sporangiospores was statistically different (group A $111 \pm 2.1$ vs group B $62 \pm 5.6, p=0.005$ ) even when both groups had levels of HbA1c $<7 \%$. It was evident that the decreasing number of sporangiospores corresponded to the starting of the germination process. The percentage of germinated sporangiospores was higher in the group A (without diabetes) after $6 \mathrm{~h}$ (group A $56 \% \pm 3$ vs group B 35\% \pm 4 , group C $48 \% \pm 4$, group D $46 \% \pm 1.4, p=0.01$ ), 


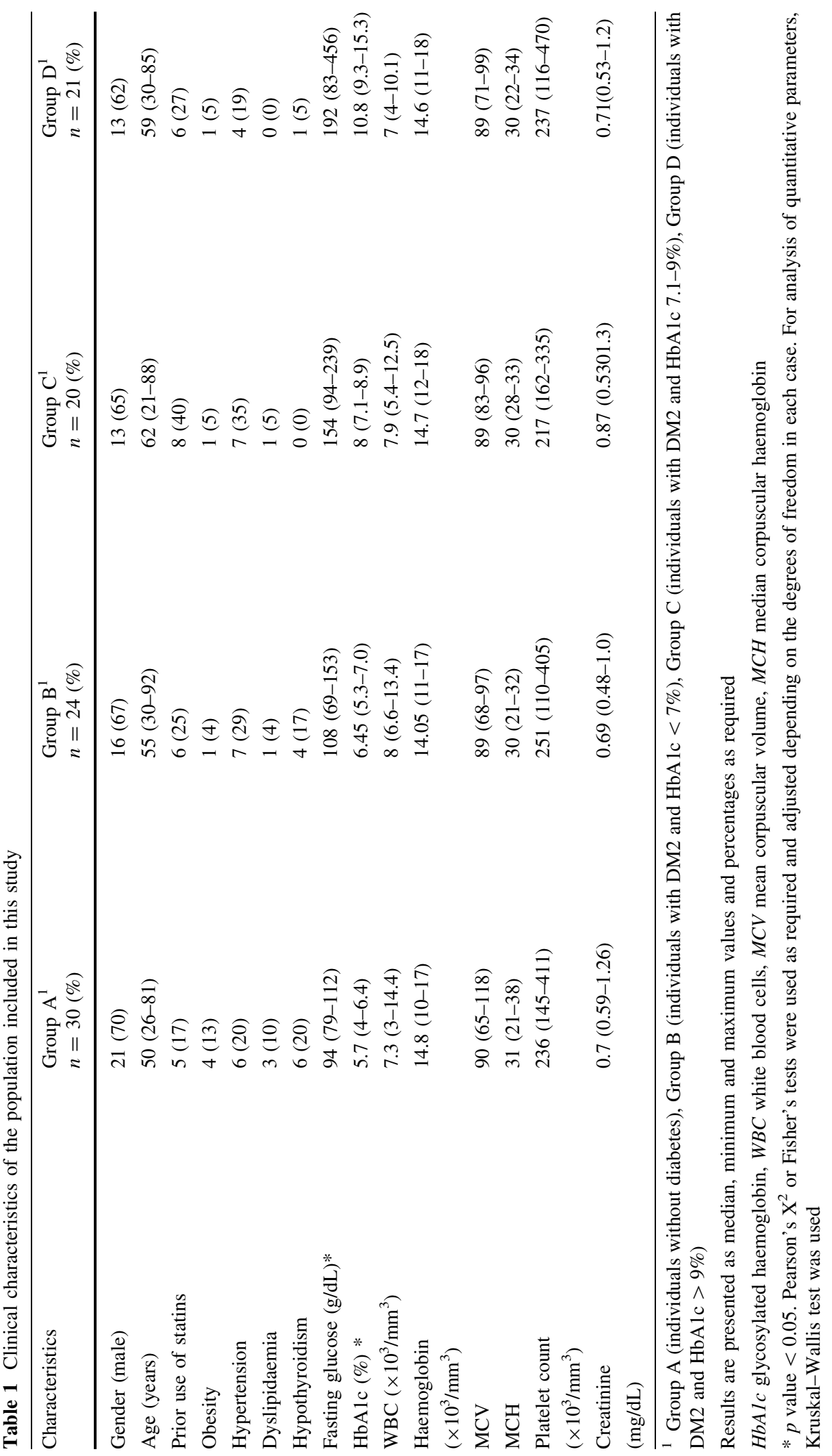


Fig. 3 Number of sporangiospores during the period of observation. Plot showing the results presented as mean and standard deviation obtained from the duplicated experiments. $N=95$. Oneway ANOVA, $* p<0.01$. Group A: individuals without diabetes. Groups $B$ (HbA1c $\leq 7 \%), C$ $(\mathrm{HbA} 1 \mathrm{c}=7.1-9 \%)$ and $D$ (HbAlc $>9 \%)$ : individuals with diabetes. The decrease in number of sporangiospores corresponds to the start of the germination process

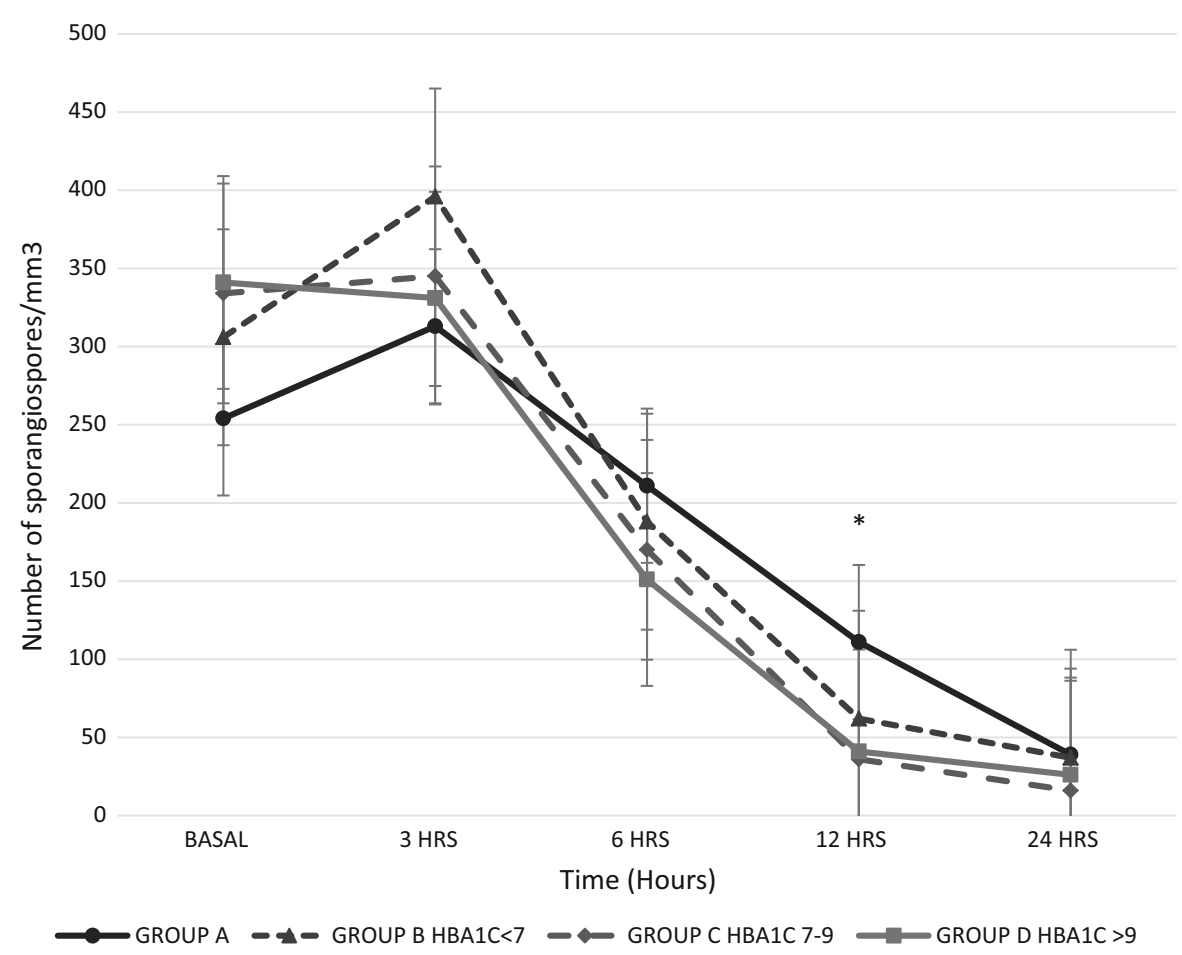

$12 \mathrm{~h}$ (group A $54 \% \pm 1.4$, group B $19 \% \pm 4$, group C $16 \% \pm 1$, group $\mathrm{D} 9.5 \% \pm 5, p<0.001)$ of incubation and was maintained until $24 \mathrm{~h}$ (group A $29 \% \pm 1$, group B $12 \% \pm 4$, group C $13.5 \% \pm 3.5$, group D $12 \% \pm 1, p<0.01$ ), as shown in Fig. 4. The more evident germination difference between groups with and without diabetes was at $12 \mathrm{~h}$ of incubation, as shown in Table S1. The lower germination rate in groups with diabetes was due to the faster hyphae formation.

The Grade of Filamentation in vitro of $R$. oryzae was Higher in all Groups with Diabetes Including Those with Glycaemic Control when Compared with the Group Without Diabetes

The grade of filamentation was higher and faster in all groups with diabetes compared with the group without diabetes after $3 \mathrm{~h}$ of incubation (Fig. 4). The group B (with diabetes and HbA1c $\leq 7 \%$ ) showed higher and faster grade of filamentation than individuals without diabetes at $6 \mathrm{~h}$ (group A $0.4 \pm 0.04$ vs group B $1 \pm 0.09, p<0.001$ ) and $24 \mathrm{~h}$ (group A $1.6 \pm 0.05$ vs group B $2.1 \pm 0.1, p<0.05$ ) (Fig. 5). All groups with diabetes had higher filamentation grade than the group A, as shown in Table S2. Between all the diabetes groups, the grade of filamentation was higher for groups B and C, as shown in Table S3. Also, when mycelial growth was evaluated in SDA, groups with diabetes initiated the growth faster than the group without diabetes at $3 \mathrm{~h}$ (group A $1 \mathrm{~mm} \pm 0.14$, group B $3 \mathrm{~mm} \pm 0.09$, group C $2.3 \mathrm{~mm} \pm 0.17$, group D $2.23 \mathrm{~mm} \pm 0.13, p<0.001)$. After $3 \mathrm{~h}$ of incubation on SDA, the growing was similar between groups being the group $\mathrm{D}$ the one with higher and faster growing out of all the other groups (Table 2).

Finally, to rule out if the $R$. oryzae growth was influenced by the use of statins, a statistical analysis of all the growing characteristics was performed and no differences were found (Table S4). On the other hand, it was noted that $R$. oryzae germination and filamentation were higher when fasting glucose levels were higher than $200 \mathrm{~g} / \mathrm{dL}$ (Table S5), and these levels were found only in groups $\mathrm{C}(\mathrm{HbA} 1 \mathrm{c}$ levels between 7.1 and 9\%) and D (HbA1c > 9\%) with diabetes (Fig. 5).

\section{Discussion}

This study describes that the beginning of filamentation process in $R$. oryzae depends on the length of germination process. In samples of the group without 
Fig. 4 Germination rate during the period of observation. Plot showing the results presented as mean and standard deviation obtained from the duplicated experiments. $N=95$, Oneway ANOVA, $* p<0.01$, $* * p<0.001$. Group A: individuals without diabetes. Groups B (HbA1c $\leq 7 \%), C$

$(\mathrm{HbA} 1 \mathrm{c}=7.1-9 \%)$ and $D$ (HbA1c $>9 \%)$ : individuals with diabetes. Decreasing the germination rate corresponds to the start of the filamentation process

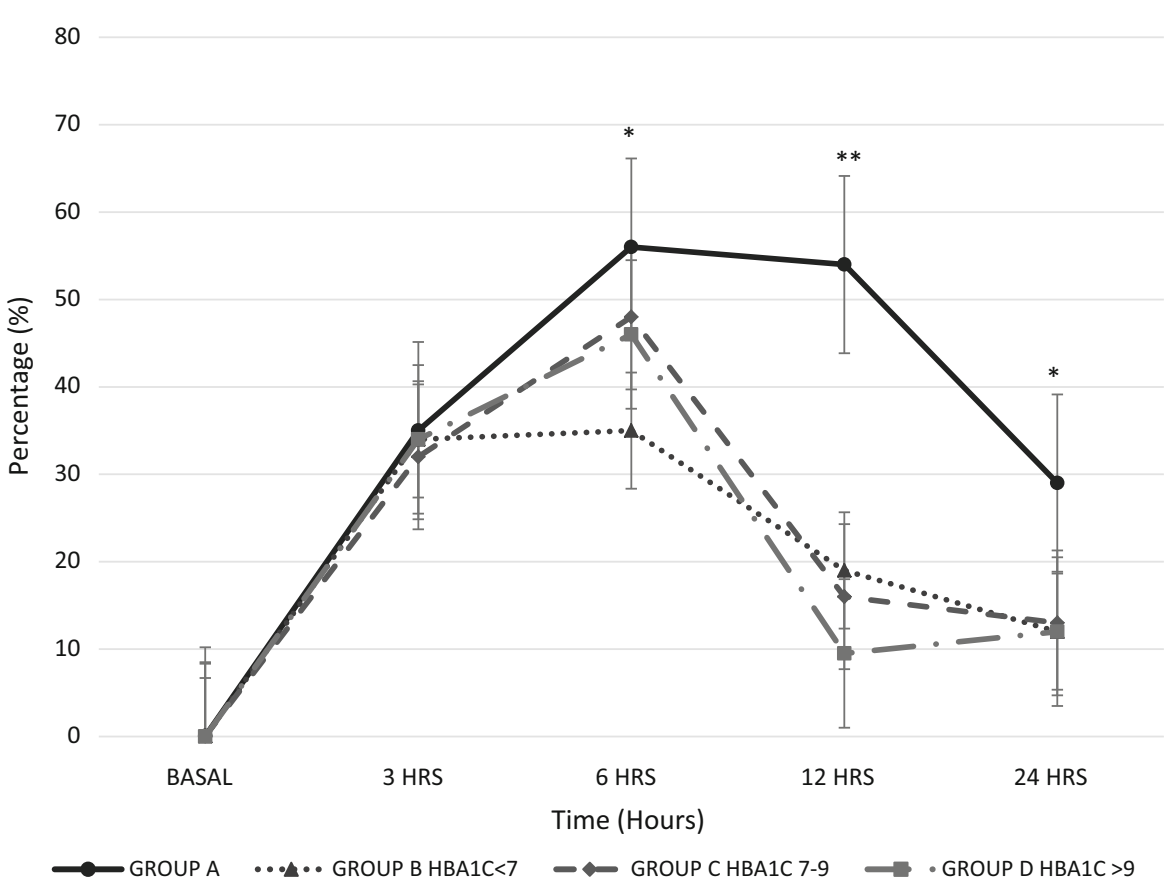

diabetes, germination period was longer, reflecting that the hyphae were developed after a longer period; meanwhile, filamentation process started earlier in blood samples of groups with uncontrolled diabetes. Interestingly, in the controlled diabetic group, the filamentation grade was lower than in the uncontrolled diabetic groups but significantly higher when compared with the non-diabetes group.

The higher growth capacity of $R$. oryzae in samples of individuals with diabetes could be explained by high concentrations of glucose and iron being the main factors favouring the germination and fungal invasion of $R$. oryzae $[10,18]$. Iron exists in two states, $\mathrm{Fe}^{2+}$ (ferrous) or $\mathrm{Fe}^{3+}$ (ferric), and the latest state is insoluble which limits its transport to the fungal intracellular compartment [18]. Iron is essential for $R$. oryzae growth, this pathogen has developed mechanisms to improve the assimilation of iron such as ferritins to store it, internal and external siderophores, and reductase/permease systems to improve iron uptake, and also iron can be obtained by $R$. oryzae from host haemoglobin [19]. In individuals with diabetic ketoacidosis, it is known that low $\mathrm{pH}$ promotes the release of iron [13]; however, hyperglycaemia, by itself, also promotes the damage of ironsequestering proteins such as haemoglobin causing the releasing of serum-free iron [20]. In this study, none of the individuals had diabetic ketoacidosis although individuals with high $\mathrm{HbA} 1 \mathrm{c}$ had also higher fasting glucose levels, meaning the main factor for the higher fungal growing was due to high levels of glucose, and probably the secondary releasing of iron due to hyperglycaemia.

The GRP78 protein has been previously reported as the receptor for CotH3 ligand in $R$. oryzae [21], allowing the interactions between the host and fungi. The expression of GRP78 protein is promoted by high concentrations of glucose, iron and acidotic environments [18]. The overexpression of GRP78 protein leads to higher rates of endocytosis and cellular damage allowing the invasion, injury and dissemination of $R$. oryzae. In our study, the cells were lysed and therefore it could not be said that the higher concentration of GRP78 protein is playing a role in the higher growth rates of $R$. oryzae in diabetic groups. 
Fig. 5 The grade of filamentation during the period of observation. Plot showing results presented as mean and standard deviation obtained from the duplicated experiments. $N=95$. Oneway ANOVA, $* p<0.001$, $* * p=0.005$. Grade of filamentation/hypha formation per 100 fields $(0=$ no hyphae in 100 fields, $+=1-100$ hyphae in 100 fields, $++=101-200$ hyphae in 100 fields, $+++=$ more than 201 hyphae in 100 fields). Group $A$ : individuals without diabetes. Groups $B$ (HbA1c $\leq 7 \%), C(\mathrm{HbA} 1 \mathrm{c}=$ $7.1-9 \%)$ and $D$

(HbAlc $>9 \%)$ : individuals with diabetes
4

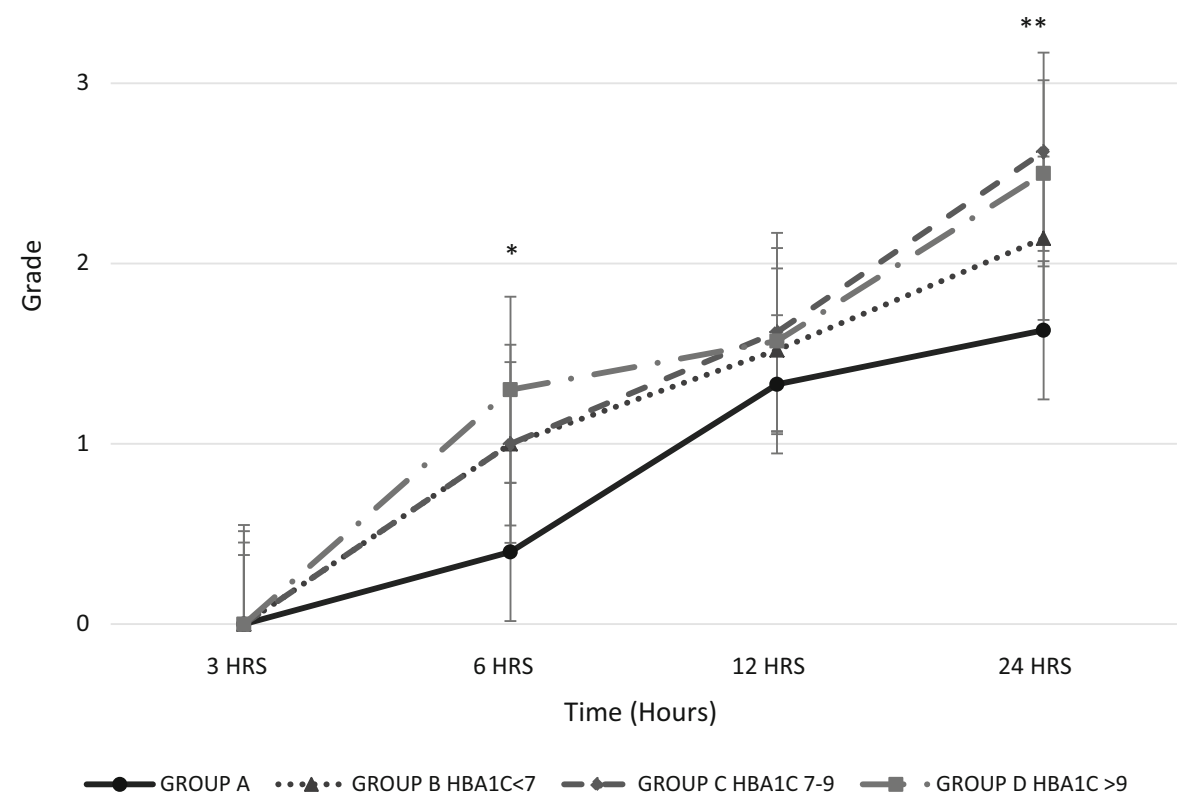

Table 2 The growth of $R$. oryzae in Sabouraud dextrose agar

\begin{tabular}{llcll}
\hline Group $(N=95)$ & $3 \mathrm{~h}(\mathrm{~mm})^{*}$ & $6 \mathrm{~h}(\mathrm{~mm})^{* *}$ & $12 \mathrm{~h}(\mathrm{~mm})^{* * *}$ & $24 \mathrm{~h}(\mathrm{~mm})$ \\
\hline A $(n=30)$ & $1.0 \pm 0.14$ & $7.8 \pm 0.7$ & $34 \pm 0.33$ & $82 \pm 0.7$ \\
B $(n=24)$ & $3.0 \pm 0.09$ & $8.7 \pm 0.8$ & $30 \pm 2.5$ & $81 \pm 2.1$ \\
C $(n=20)$ & $2.3 \pm 0.17$ & $9.4 \pm 0.2$ & $32 \pm 0.56$ & $83 \pm 0.7$ \\
D $(n=21)$ & $2.2 \pm 0.13$ & $11.0 \pm 0.8$ & $34 \pm 0.14$ & $81 \pm 1.4$ \\
\hline
\end{tabular}

The results are presented as mean and standard deviation obtained from the duplicated experiments. Group A: individuals without diabetes. Group B $(\mathrm{HbA} 1 \mathrm{c} \leq 7 \%), \mathrm{C}(\mathrm{HbA} 1 \mathrm{c}=7.1-9 \%)$ and $\mathrm{D}(\mathrm{HbA} 1 \mathrm{c}>9 \%)$ are individuals with diabetes. Mm $=$ millimetres. $P$ values shown in this table were calculated with Fisher's LSD test

A versus B, both groups had HbA1c $<7 \%$, but group A had not diabetes and group B had diabetes, * $p=0.0001, * * * p=0.03$

$\mathrm{B}$ versus $\mathrm{C}$, groups with diabetes with $\mathrm{HbA1c}<7 \%$ versus $7.1-9 \%, * p=0.005$

$\mathrm{B}$ versus $\mathrm{D}$, groups with diabetes with $\mathrm{HbA1c}<7 \%$ versus $>9 \%, * p=0.003$, ** $p=0.02$, *** 0.04

$\mathrm{C}$ versus $\mathrm{D}$, groups with diabetes with $\mathrm{HbA1c} 7-9 \%$ versus $>9 \%, p=\mathrm{NS}$

The main observation in this study was that despite the glycaemic control, diabetic population could still be at risk of mucormycosis and, hence, have worst clinical outcomes. Tuberculosis is one example of disease where glycaemic control decreases the rates of infection but not at non-diabetic population rates. The incidence of tuberculosis could be twice higher in individuals with controlled diabetes compared with individuals without diabetes [22]. Similar observations have been also reported for urinary tract infections [23] and major macrovascular complications [24].

On the other hand, the role of HbA1c as the best marker of glycaemic control has been debated because it correlates with chronic sustained hyperglycaemia but not with all the acute glucose variability such as postprandial hyperglycaemia excursions which are best evaluated with mean amplitude of glycaemic excursion [25]. This implies that individuals with controlled diabetes in our study, although having 
normal fasting glucose levels, could be experiencing hyperglycaemic postprandial periods in a regular basis, which are not experienced by non-diabetic individuals. These hyperglycaemic episodes could be having indirect effects on releasing of free serum iron, and therefore, this mechanism would be favouring the $R$. oryzae growth in our assays. Unfortunately, the measurement of free iron was not taken in our study due to its original design. The impact of this glycaemic variability has been previously associated with higher risk of sepsis and mortality in individuals suffering burns and autologous haematopoietic cell transplant recipients [26, 27].

The findings in this study require to be studied and described in greater depth. In the future, interactions with host will be evaluated by our group using in vitro and in vivo models. At the authors' knowledge, this is the first study reporting individuals with diabetes and good glycaemic control could still be at higher risk of mucormycosis than the non-diabetic individuals. These findings could partially answer and lead to further research of why more than $50 \%$ of the mucormycosis cases are presented without diabetic ketoacidosis [2] and why some cases have chronic evolution, instead of acute presentation [28], without an apparently associated factor with this clinical feature.

\section{Conclusion}

Individuals with diabetes and good glycaemic control had lesser and slower in vitro proliferation of $R$. oryzae compared with uncontrolled ones; however, it was still higher than the individuals without diabetes. The main observation in this study was that despite the glycaemic control, diabetic population might have a higher risk of developing mucormycosis.

Acknowledgement We thank all members of the Laboratory of Clinical Microbiology in the Hospital General Dr. Manuel Gea González, Instituto Nacional de Rehabilitacion and Hospital General de México. Also, thanks to the Wellcome Trust Strategic Award, corresponding author's scholarship sponsor.

Financial Support This study did not have pharmaceutical or grant support, and resources were obtained from institutional budgets.

\section{Compliance with Ethical Standards}

Conflict of interest Authors do not have conflict of interest in this study. RSJ, FCR and RZAP have been speakers and consultants for Pfizer, MSD, Stendhal. The information content in this study was partially presented during the 26th ECCMID in April 2016, Amsterdam, The Netherlands.

Open Access This article is distributed under the terms of the Creative Commons Attribution 4.0 International License (http:// creativecommons.org/licenses/by/4.0/), which permits unrestricted use, distribution, and reproduction in any medium, provided you give appropriate credit to the original author(s) and the source, provide a link to the Creative Commons license, and indicate if changes were made.

\section{References}

1. Skiada A, Pagano L, Groll A, Zimmerli S, Dupont B, Lagrou K, et al. Zygomycosis in Europe: analysis of 230 cases accrued by the registry of the European Confederation of Medical Mycology (ECMM) Working Group on Zygomycosis between 2005 and 2007. Clin Microbiol Infect. 2011;17(12):1859-67.

2. Roden MM, Zaoutis TE, Buchanan WL, Knudsen TA, Sarkisova TA, Schaufele RL, et al. Epidemiology and outcome of zygomycosis: a review of 929 reported cases. Clin Infect Dis [Internet]. 2005;41(5):634-53.

3. Vaezi A, Moazeni M, Rahimi MT, de Hoog S. Badali H. Mucormycosis in Iran: A systematic review. mycoses; 2016. p. 402-15.

4. Kennedy KJ, Daveson K, Slavin MA, van Hal SJ, Sorrell TC, Lee A, et al. Mucormycosis in Australia: contemporary epidemiology and outcomes. Clin Microbiol Infect. 2016;22(9):775-81.

5. Lelievre L, Garcia-Hermoso D, Abdoul H, Hivelin M, Chouaki T, Toubas D, et al. Posttraumatic mucormycosis. Med (Baltimore) [Internet]. 2014;93(24):373-82.

6. Lanternier F, Dannaoui E, Morizot G, Elie C, Garcia-Hermoso D, Huerre M, et al. A global analysis of mucormycosis in France: the RetroZygo study (2005-2007). Clin Infect Dis. 2012;54(SUPPL. 1):35-43.

7. Sims CR, Ostrosky-Zeichner L. Contemporary treatment and outcomes of zygomycosis in a non-oncologic tertiary care center. Arch Med Res. 2007;38(1):90-3.

8. Turunc T, Demiroglu YZ, Aliskan H, Colakoglu S, Arslan $\mathrm{H}$. Eleven cases of mucormycosis with atypical clinical manifestations in diabetic patients. Diabet Res Clin Pract. 2008;82(2):203-8.

9. Camara-Lemarroy CR, González-Moreno EI, RodríguezGutiérrez R, Rendón-Ramírez EJ, Ayala-Cortés AS, FragaHernández ML, et al. Clinical features and outcome of mucormycosis. Interdiscip Perspect Infect Dis. 2014; 2014:562610.

10. Waldorf AR, Ruderman N, Diamond RD. Specific susceptibility to mucormycosis in murine diabetes and 
bronchoalveolar macrophage defense against Rhizopus. J Clin Invest. 1984;74(1):150-60.

11. Ibrahim AS, Kontoyiannis DP. Update on mucormycosis pathogenesis. Curr Opin Infect Dis. 2013;26(6):508-15.

12. Shirazi F, Farmakiotis D, Yan Y, Albert N, Do KA, Kontoyiannis DP. Diet modification and metformin have a beneficial effect in a fly model of obesity and mucormycosis. PLoS ONE. 2014;9(9):1-9.

13. Gebremariam T, Lin L, Liu M, Kontoyiannis DP, French S $\mathrm{Jr}$, EE J, et al. Bicarbonate correction of ketoacidosis alters host- pathogen interactions and alleviates mucormycosis. J Clin Invest. 2016;126(6):1-15.

14. Cornely OA, Arikan-Akdagli S, Dannaoui E, Groll AH, Lagrou K, Chakrabarti A, et al. ESCMID and ECMM joint clinical guidelines for the diagnosis and management of mucormycosis 2013. Clin Microbiol Infect. 2014; 20(S3):5-26.

15. Kontoyiannis DP, Lewis RE. How I treat mucormycosis. Blood. 2011;118(5):1216-24.

16. Torjesen I. NEWS NICE recommends tighter blood sugar control in diabetes to reduce risk of complications. BMJ. 2015;351:h4612.

17. Irinyi L, Lackner M, de Hoog GS, Meyer W. DNA barcoding of fungi causing infections in humans and animals. Fungal Biol. 2016;120(2):125-36.

18. Liu M, Spellberg B, Phan QT, Fu Y, Fu Y, Lee AS, et al. The endothelial cell receptor GRP78 is required for mucormycosis pathogenesis in diabetic mice. J Clin Invest. 2010;120(6):1914-24.

19. Ibrahim A, Spellberg B, Edwards JJ. Iron Acquisition: a novel prospective on mucormycosis pathogenesis and treatment. Curr Opin Infect Dis. 2008;21(6):620-5.

20. Kar M, Chakraborti a S. Release of iron from haemoglobina possible source of free radicals in diabetes mellitus. Indian J Exp Biol [Internet]. 1999;37(2):190-2.
21. Gebremariam T, Liu M, Luo G, Bruno V, Phan QT, Waring $\mathrm{AJ}$, et al. CotH3 mediates fungal invasion of host cells during mucormycosis. J Clin Invest. 2014;124(1):237-50.

22. Lo HY, Yang SL, Lin HH, Bai KJ, Lee JJ, Lee TI, et al. Does enhanced diabetes management reduce the risk and improve the outcome of tuberculosis? Int $J$ Tuberc Lung Dis. 2016;20(3):376-82.

23. Lenherr SM, Clemens JQ, Braffett BH, Cleary PA, Dunn RL, Hotaling JM, et al. Glycemic control and urinary tract infections in women with type 1 diabetes: results from the DCCT/EDIC. J Urol. 2016;196:1129-35.

24. Kohnert K-D, Heinke P, Vogt L, Salzsieder E. Utility of different glycemic control metrics for optimizing management of diabetes. World J Diabetes [Internet]. 2015;6(1): $17-29$.

25. Kohnert KD, Augstein P, Heinke P, Zander E, Peterson K, Freyse EJ, et al. Chronic hyperglycemia but not glucose variability determines $\mathrm{HbA} 1 \mathrm{c}$ levels in well-controlled patients with type 2 diabetes. Diabetes Res Clin Pract. 2007;77(3):420-6.

26. Pisarchik AN, Pochepen ON, Pisarchyk LA. Increasing blood glucose variability is a precursor of sepsis and mortality in burned patients. PLoS ONE. 2012;7(10):1-8.

27. Hammer MJ, Melkus GD, Knobf MT, Casper C, Fletcher J, Cleland CM. Glycemic status and infection risk in nondiabetic autologous hematopoietic cell transplantation recipients. Biol Res Nurs [Internet]. 2016;18(3):344-50.

28. Gutiérrez-Delgado EM, Treviño-González JL, Montemayor-Alatorre A, Ceceñas-Falcón LA, Ruiz-Holguín E, Andrade-Vázquez CJ, et al. Chronic rhino-orbito-cerebral mucormycosis: a case report and review of the literature. Ann Med Surg [Internet]. 2016;6:87-91. 